\section{§11. Conceptual Design of an Indirect-cooled Superconducting Magnet for the LHD- type Fusion Reactor FFHR}

Takahata, K., Mito, T., Tamura, H., Imagawa, S., Sagara, A.

Heliotron power reactors have competitive advantages for steady-state operation due to the fact that they employ currentless plasma. These advantages have been demonstrated by the Large Helical Device (LHD) with a superconducting magnet system since its development nine years ago. Engineering and physics results from studies of the LHD confirm that LHD-type heliotron reactors are well suited for steady-state power plants.

Methods for cooling a superconducting coil are classified into three types: pool cooling, forced-flow cooling and indirect cooling. The helical coils of the LHD are pool cooled with liquid helium. Recently developed superconductors for use in fusion reactors are mostly forced-flow-cooled types, including the conductors for the LHD poloidal field coils, the W7-X and ITER. Forced-flow-cooled conductors have higher rigidity and greater dielectric strength than pool-cooled conductors. However, forced-flow-cooled conductors require a circulating pump to compress cryogenic helium gas to overcome the pressure drop across the coil. For a large coil, the pressure drop limits the unit conductor length, and a large number of pipes and conductor joints must be installed. 'Indirect cooling' is a promising candidate for fusion magnets because it solves the issue of the pressure drop. ${ }^{1)}$ However, no indirect-cooled coils have been constructed for fusion reactors, even though indirect cooling is commonly used in accelerator magnets. In this study, we therefore presents a design of a superconducting helical coil of the LHD-type fusion reactor FFHR using an indirect-cooled superconductor with $\mathrm{Nb}_{3} \mathrm{Sn}$. Feasibility studies have been performed from the perspective of eliminating heating of the superconductor due to nuclear heating, support of an electromagnetic force and quench protection.

Fig. 1 illustrates the magnet system for the FFHR. The magnet system consists of a pair of helical coils and two pairs of poloidal field coils. All the coils are built to withstand electromagnetic forces by inner and outer supporting structures. ${ }^{2)}$ The ideal cross section of the helical coils was determined by considering the radial build of a blanket. Fig. 2 shows the cross-section and structure of the helical coil. The preliminary design provides a rectangular cross-section ( $1.8 \mathrm{~m}$ wide and $0.9 \mathrm{~m}$ high). The $100 \mathrm{kA}$ superconductor is wound by a layer winding method (36 turns/layer, 12 layers). Four 75-mm-thick cooling panels are installed inside the coil. Cooling channels, in which helium coolant flows, are embedded in the panels. The superconductor is indirectly cooled by heat conduction in the cross-sectional direction. The coil is then covered with a stainless steel coil case. In the conductor, a bundle of superconducting and copper wires is embedded in an aluminum-alloy jacket with a high filling factor. ${ }^{1)}$ Commercial $\mathrm{Nb}_{3} \mathrm{Sn}$ wires can be used because they are widely used at $13 \mathrm{~T}$, the maximum field of the FFHR. The use of an aluminum-alloy jacked superconductor is novel because the melting point of aluminum $(933 \mathrm{~K})$ is a little lower than the heat treatment temperature of $\mathrm{Nb}_{3} \mathrm{Sn}$ wires $(\sim 1000 \mathrm{~K})$. Therefore the jacketing must be performed after the heat treatment of the wires. We developed a conductor fabrication process using a recently developed friction stir welding (FSW) technique that uses friction heating.

The feasibility studies provide the following results: (1) The cooling panels in the coil can eliminate steady-state nuclear heating and control the temperature increase. (2) The helical coil with a stainless-steel coil case can withstand the radial electromagnetic force. (3) The coil can be protected against damage due to an unexpected quench by dividing the coil into subdivisions. These results confirm that the indirect-cooled coil is a promising candidate for fusion magnets.

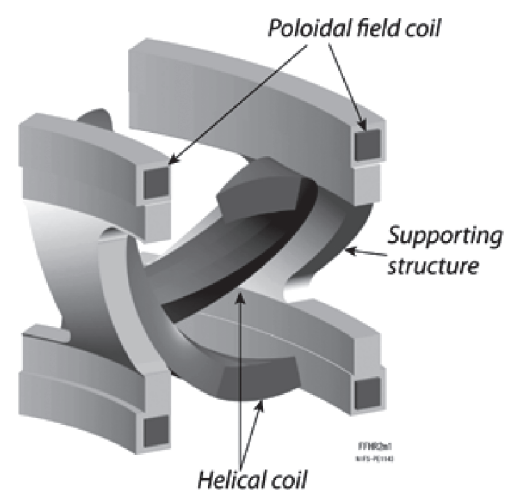

Fig. 1. Schematic illustration of the FFHR magnet system (36-degree segment).

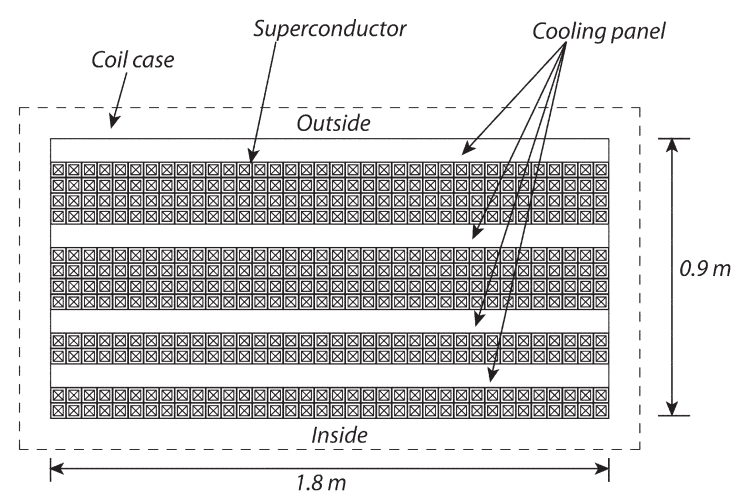

Fig. 2. Structure of the helical coil.

\section{Reference}

1) Mito T., et al., Fusion Eng. Des. 81 (2006) 2389

2) Sagara A., et al., Nucl. Fusion 45 (2005) 258 